\title{
Dual Role of Mitophagy in Cancer Drug Resistance
}

\author{
CHEN YAN and TAO-SHENG LI
}

Department of Stem Cell Biology, Nagasaki University Graduate School of Biomedical Sciences, Sakamoto, Japan

\begin{abstract}
The effectiveness of chemotherapy is largely limited by drug resistance. In the past few decades, modulation of anticancer drug resistance plays little role in benefit of clinical outcomes due to complex drug resistance mechanisms. Mitophagy, an important mitochondrial quality control system, selectively degrades excessive or damaged mitochondria by autophagy. Accumulating reports are suggesting that dysregulation of mitophagy contributes to neoplastic progression and drug resistance in various types of tumors. Mitophagy was originally thought to be an oncosuppressor that maintains cellular homeostasis and prevents oncogenic transformation. On the other hand, mitophagy promotes cancer cell survival under cytotoxic stress by degrading damaged mitochondria and reducing mitochondrial reactive oxygen species. Therefore, induction and inhibition of mitophagy in cancer drug resistance are controversial. In this review, we summarize current knowledge on the dual role of mitophagy in cancer drug resistance.
\end{abstract}

Although chemotherapy is one of the most widely used strategies for cancer therapy, its effectiveness is limited by drug resistance $(1,2)$. Complex mechanisms mediate drug resistance in cancer, such as alterations in the drug target, activation of pro-survival pathways and ineffective induction of cell death (2). In the past few decades, modulation of anticancer drug resistance has played little role in the benefit of clinical outcomes (3). Therefore, there is an urgent demand for enhancing anticancer drug sensitivity via discovery of new appropriate targets.

This article is freely accessible online.

Correspondence to: Tao-Sheng Li, MD, Ph.D., Department of Stem Cell Biology, Nagasaki University Graduate School of Biomedical Sciences, 1-12-4 Sakamoto, Nagasaki 852-8523, Japan. Tel: +81 958197097, Fax: +81 958197100, e-mail: litaoshe@ nagasaki-u.ac.jp

Key Words: Mitophagy, neoplasms, drug resistance, chemotherapy, review.
Mitophagy, a selective autophagic process, has been shown to promote tumorigenesis and cell survival in various types of tumors by removing abnormal mitochondria $(4,5)$. The toxicity of most chemotherapeutic agents is partially attribute to the induction of mitochondrial dysfunction and oxidative stresses $(6,7)$. Thus, the rapid clearance of damaged mitochondria by mitophagy is thought to mediate drug resistance in cancer cells (5). In addition, excessive mitochondrial clearance may induce cell metabolic disorders and cell death (8). Therefore, mitophagy likely plays a dual role in cancer drug resistance depending on different conditions and cell types. In this review, we focus on current research efforts to identify and overcome underlying mechanisms of mitophagy-related drug resistance.

\section{Biological Role of Mitophagy}

Mitochondria are crucial organelles for energy metabolism, regulation of cell signaling and apoptosis in mammalian cells (9). In order to maintain cellular homeostasis, the cell has evolved complex systems for the quality control of mitochondria. One of these systems, mitophagy, selectively degrades excessive or damaged mitochondria by autophagy in response to various stresses (Figure 1) $(10,11)$. Autophagy is an essential catabolic pathway that degrades proteins or other cellular components within lysosomes (12). Differently from canonical autophagy, mitophagy selectively targets mitochondria which have been marked by mitophagy receptors. These multiple receptor systems mainly include Parkin/PTEN-induced putative kinase 1 (PINK1) receptor system, microtubuleassociated proteins 1 light chain 3 (LC3)-interacting region (LIR)-containing receptor system [e.g. B-cell lymphoma 2 (BCL2)-interacting protein 3 (BNIP3) and BNIP3-like (BNIP3L)] and lipid-mediated system (e.g. ceramide) $(13,14)$.

Autophagy can act as a survival mechanism for cancer cells in response to various stresses (15-17), but the role of canonical autophagy in cancer remains unclear $(5,18,19)$. Therefore, how about mitophagy in cancer? Mitophagy is causally linked to various physiological processes and diseases, for example defective mitophagy will impair 
survival of memory natural killer cells, conferring Parkinson's disease and cardiac defects $(13,20,21)$. Furthermore, evidence indicates that dysfunction of mitophagy also promotes tumorigenesis and neoplastic progression $(4,5,14)$. Guo et al. reported that the induction of mitophagy in macrophages prevents the progression of colitis-associated cancer (22). Moreover, tumorigenesis and metastasis are also promoted by mitochondrial reactive oxygen species (ROS) (23). According to Chourasia et al., BNIP3 loss, and ensuing defects in mitophagy, leads to ROS production and mammary neoplastic progression to metastasis (4).

\section{Manipulating Mitophagy as a Potential Target for Cancer Therapy}

Autophagy regulators (e.g. chloroquine, hydrochloroquine) have entered dozens of clinical studies on anticancer therapy (See https://clinicaltrials.gov/ct2/results?term=autophagy+ and+cancer). There is great interest in manipulating mitophagy in order to improve cancer therapy, while these strategies are considerably confusing $(5,24,25)$. The functional outcome of mitophagy-induced cell death or survival dependent on cancer therapy relies on different therapeutic treatments and cell types (Figure 2).

Induction of mitophagy increases cancer cell death and chemotherapy sensitivity. Ceramide, a central molecule of sphingolipid metabolism, is involved in the regulation of mitophagy (26). Studies show that CerS1/C18-ceramide selectively mediates lethal mitophagy, which is B-cell lymphoma 2-associated X (BAX)/BCL2 antagonist/killer 1 (BAK1)- and caspase-independent $(24,27)$. Ceramide induces the formation of lipidated LC3, which then binds ceramide on the mitochondrial membrane upon dynaminrelated protein 1 (DRP1)-mediated mitochondrial fission, targeting autophagolysosomes to mitochondria and leading to lethal mitophagy in cancer cells $(24,27)$. Excessive mitochondrial clearance in the absence of mitochondrial biogenesis or metabolic plasticity likely induce cell metabolic disorders and death in cancer cells (8). Moreover, DRP1-mediated fission is also a prerequisite for mitophagy induced by BNIP3, which is a protective response activated by cardiac myocytes (28). Understanding the underlying mechanism for different effects of DRP1 between cancer cells and cardiac myocytes may help in better anticancer drug formulation.

FMS-like tyrosine kinase 3 (FLT3) is a membrane-bound receptor tyrosine kinase expressed by immature hematopoietic cells (29). Mutations of FLT3 have been detected in about $30 \%$ of patients with acute myelogenous leukemia (AML), and the most common activating FLT3 mutation is an internal tandem duplication (ITD) in the juxtamembrane domain (FLT3-ITD) (29). FLT3-ITD inhibitors, such as sorafenib, quizartinib
(AC220), and crenolanib, showed efficacy for therapy in preclinical models of AML, but not in clinical trials because of the development of drug resistance (30). Interestingly, LCL461, a mitochondria-targeted ceramide analog drug, is efficacious in attenuating crenolanib resistance by inducing lethal mitophagy in FLT3-ITD ${ }^{+}$AML in vitro and in vivo (27). This lethal pathway of mitophagy induction by ceramide and ceramide analog drug is likely be a potential target in FLT3ITD+ AML, and further studies will be needed to determine if ceramide will be effective in other cancer types.

Inhibition of mitophagy enhances drug sensitivity. A great number of studies have demonstrated that mitophagy can be inhibited through genetically or pharmacologically targeting different stages of the autophagic/mitophagic process, such as silencing of autophagic/mitophagic genes (e.g. autophagyrelated 5 (ATG5), Parkin, PINK1, BNIP3 and BNIP3L) (5, 25, 31 ), blocking the formation of autophagosomes by phosphoinositide 3-kinase (PI3K) inhibitors (e.g. 3-methyladenine and LY294002) $(25,31)$ and inhibiting the fusion of autophagosomes with lysosomes or the degradation capacity of autophagolysosomes (e.g. chloroquine, bafilomycin A1, leupeptin and liensinine) $(25,31,32)$.

Classical inhibitors of mitophagy. Doxorubicin, a DNAdamaging agent, is believed to cause toxicity by inducing mitochondrial dysfunction and enhancing superoxide formation $(33,34)$. Our previous study found that expression of BNIP3L, a regulator of mitophagy, significantly increased in colorectal cancer stem cells after doxorubicin treatment (5). The inhibition of mitophagy by $B N I P 3 L$ silencing significantly enhanced doxorubicin sensitivity in colorectal cancer stem cells (5). But the mechanism for the regulation of BNIP3L in cancer stem cells is still unclear.

Salinomycin, an antibacterial and coccidiostat ionophore drug, also has a novel anticancer role through mitochondrial hyperpolarization (35). Mitochondrial dynamic fusion and fission mechanisms contribute to alterations in mitochondrial mass and maintenance of mitochondrial function in salinomycin-treated cells. The most important pro-survival mechanism is mitophagy, which eliminates salinomycininduced dysfunctional mitochondria. Dysfunctional mitochondria may actually consume ATP, and generate excessive amounts of harmful ROS, thus contributing to loss of cellular homeostasis. When combined with mitophagy inhibition by ATG5 knockout, toxicity of salinomycin towards cancer cells and cancer stem cells was enhanced (35).

A variety of potential drugs are currently being tested for anticancer treatments. Mitophagy inhibition in combination with some potential anticancer drugs also increases cytotoxicity. UNBS1450 is a sodium channel antagonist potentially for the treatment of cancer. In stromal neuroblastoma SH-N-AS cell line, efficient mitophagy is the 

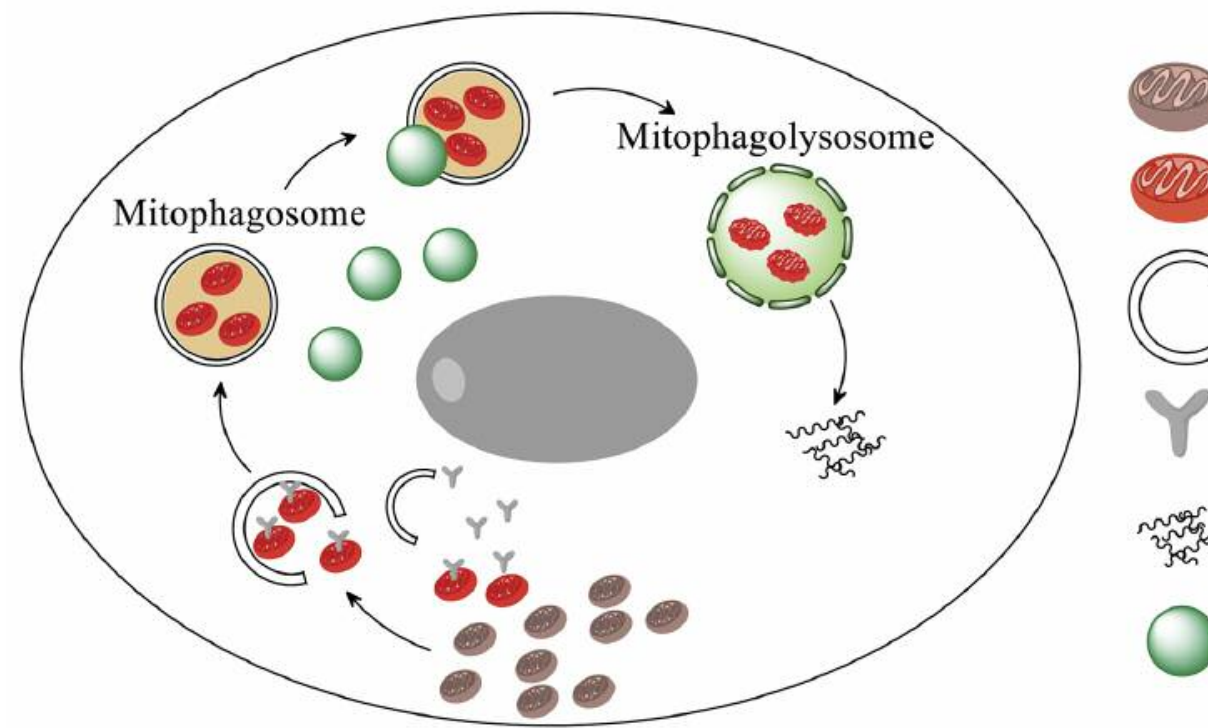

Healthy mitochondrion

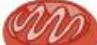

Damaged mitochondrion

$($ Mitophagic vacuole

Mitophagy receptor

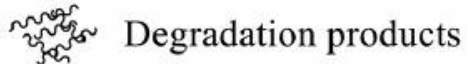

Lysosome

Figure 1. The process of mitophagy in mammalian cells.

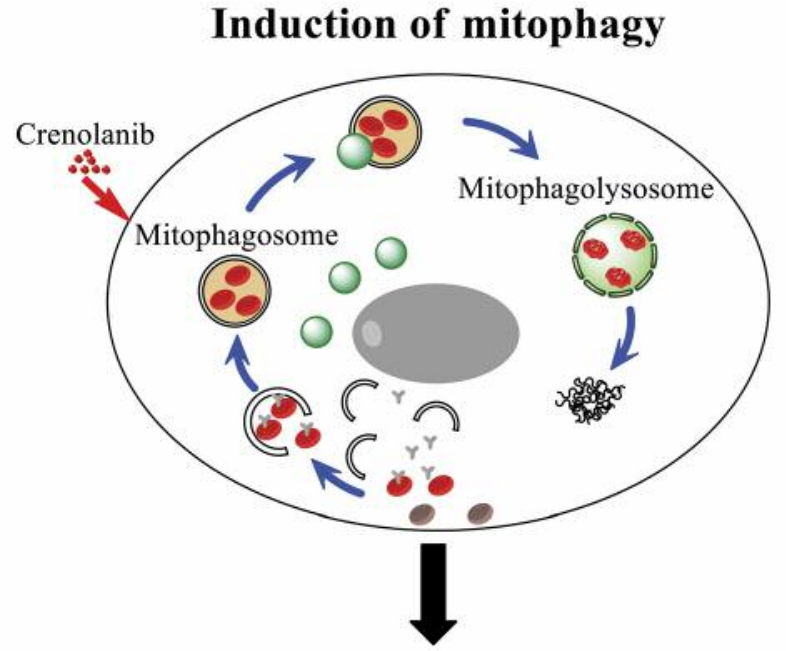

Cell death

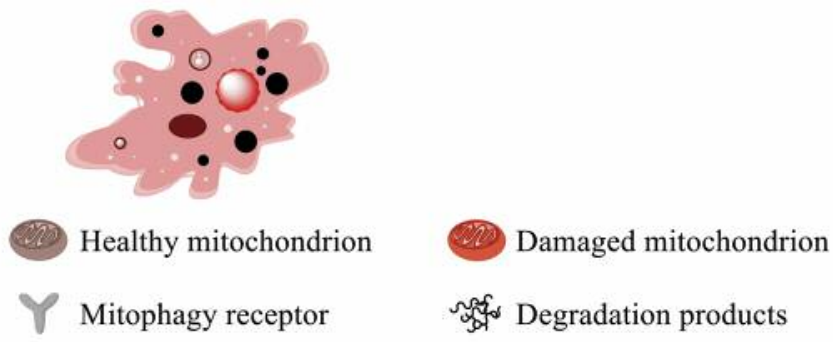

ß
Inhibition of mitophagy

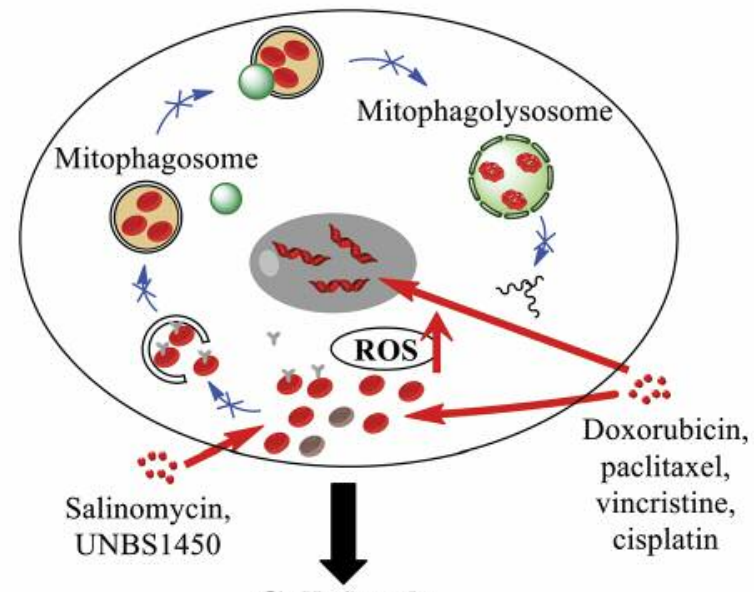

Cell death

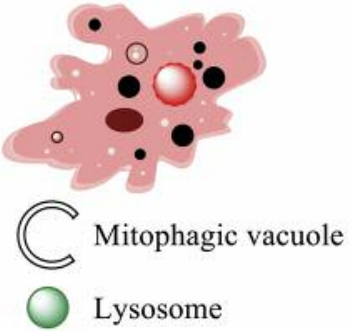

Figure 2. The controversial role of mitophagy in cancer drug resistance. 
key mechanism causing the failure of activation of the apoptotic pathway, which increased resistance to UNBS 1450 (32). Inhibition of autophagy by small inhibitory RNAs targeting ATG5, autophagy related 7 (ATG7) and Beclin-1 sensitized SK-N-AS cells by reactivating apoptosis (32).

Novel inhibitors of mitophagy. Liensinine, an isoquinoline alkaloid, is extracted from the seed embryo of Nelumbo nucifera Gaertn. The mitophagy-inhibitory effects of liensinine enhance sensitivity of breast cancer cells to classical chemotherapeutic drugs (e.g. doxorubicin, paclitaxel, vincristine, and cisplatin) (25). Liensinine induced the accumulation of mitophagosomes by inhibiting autophagosome-lysosome fusion (25). Although the effect of liensinine was similar to that of chloroquine, hydroxychloroquine, and bafilomycin A1, the mechanism of liensinine-mediated blockade of autophagosome-lysosome fusion was different (25). The latter agents alkalinized the lysosomal $\mathrm{pH}$ to suppress the fusion between with autophagosomes and interfere with the action of lysosomal hydrolases (25). However, the lysosomal $\mathrm{pH}$ was not changed in response to liensinine treatment, suggesting that alteration of lysosomal $\mathrm{pH}$ is not necessary for inhibition of autophagosomelysosome fusion and cathepsin maturation mediated by liensinine (25). The small GTP-binding protein ras-related protein 7A (RAB7A) has a role in the late endocytic pathway, lysosome biogenesis, and the final maturation of late autophagic vacuoles (36). Liensinine reduced recruitment of RAB7A to lysosomes, thus inhibiting the endocytic pathway-dependent cathepsin transport to lysosomes, and blocking autophagosome-lysosome fusion (25). It is likely one potential mechanism through which the inhibition of autophagy/mitophagy by liensinine sensitizes tumor cells to doxorubicin-induced apoptosis.

\section{Conclusion and Perspectives}

Although there was no consensus that mitophagy acts either as suppressor or inducer of cancer drug resistance in specific cancer types, the most appropriate treatment for each patient depending on the cancer type and anticancer drug can be selected. Whilst the induction of mitophagy by ceramide contributes to sensitivity to FLT3-ITD inhibitors in FLT3ITD $^{+}$AML, further studies will be needed to determine if ceramide is effective in other cancer types. The inhibition of mitophagy is likely more suitable for combination with chemotherapeutic agents in various types of cancer. Further studies are warranted to elucidate how mitophagy is regulated in different cancer types and in anticancer drug treatments, which may enlighten the development of novel strategies for the treatment of cancer.

\section{Conflicts of Interest}

The Authors declare no conflict of interest in regard to this article.

\section{Acknowledgements}

This study was supported, in part, by a Grant-in-Aid from the Ministry of Education, Culture, Sports, Science and Technology, Japan, and the Collaborative Research Program of the Atomic-bomb Disease Institute of Nagasaki University. The funder played no role in the study design, the data collection and analysis, decision to publish, or preparation of the article.

\section{References}

1 Tredan O, Galmarini CM, Patel K and Tannock IF: Drug resistance and the solid tumor microenvironment. J Natl Cancer Inst 99: 1441-1454, 2007.

2 Holohan C, Van Schaeybroeck S, Longley DB and Johnston PG: Cancer drug resistance: an evolving paradigm. Nat Rev Cancer 13: 714-726, 2013.

3 Fojo T and Bates S: Strategies for reversing drug resistance. Oncogene 22: 7512-7523, 2003.

4 Chourasia AH, Tracy K, Frankenberger C, Boland ML, Sharifi MN, Drake LE, Sachleben JR, Asara JM, Locasale JW, Karczmar GS and Macleod KF: Mitophagy defects arising from BNIP3 loss promote mammary tumor progression to metastasis. EMBO Rep 16: 1145-1163, 2015.

5 Yan C, Luo L, Guo CY, Goto S, Urata Y, Shao JH and Li TS: Doxorubicin-induced mitophagy contributes to drug resistance in cancer stem cells from HCT8 human colorectal cancer cells. Cancer Lett 388: 34-42, 2017.

6 Scatena R, Bottoni P, Botta G, Martorana GE and Giardina B: The role of mitochondria in pharmacotoxicology: a reevaluation of an old, newly emerging topic. Am J Physiol Cell Physiol 293: C12-21, 2007.

7 Fulda S, Galluzzi L and Kroemer G: Targeting mitochondria for cancer therapy. Nat Rev Drug Discov 9: 447-464, 2010.

8 Kubli DA and Gustafsson AB: Mitochondria and mitophagy: the yin and yang of cell death control. Circ Res 111: 1208-1221, 2012.

9 Vyas S, Zaganjor E and Haigis MC: Mitochondria and Cancer. Cell 166: 555-566, 2016.

10 Youle RJ and Narendra DP: Mechanisms of mitophagy. Nat Rev Mol Cell Biol 12: 9-14, 2011.

11 Zhu J, Wang KZ and Chu CT: After the banquet: mitochondrial biogenesis, mitophagy, and cell survival. Autophagy 9: 16631676, 2013

12 Galluzzi L, Pietrocola F, Bravo-San Pedro JM, Amaravadi RK, Baehrecke EH, Cecconi F, Codogno P, Debnath J, Gewirtz DA, Karantza V, Kimmelman A, Kumar S, Levine B, Maiuri MC, Martin SJ, Penninger J, Piacentini M, Rubinsztein DC, Simon HU, Simonsen A, Thorburn AM, Velasco G, Ryan KM and Kroemer G: Autophagy in malignant transformation and cancer progression. Embo J 34: 856-880, 2015.

13 Hamacher-Brady A and Brady NR: Mitophagy programs: mechanisms and physiological implications of mitochondrial targeting by autophagy. Cell Mol Life Sci 73: 775-795, 2016.

14 Bernardini JP, Lazarou M and Dewson G: Parkin and mitophagy in cancer. Oncogene 36: 1315-1327, 2017.

15 Apel A, Herr I, Schwarz H, Rodemann HP and Mayer A: Blocked autophagy sensitizes resistant carcinoma cells to radiation therapy. Cancer Res 68: 1485-1494, 2008. 
16 Jarauta V, Jaime P, Gonzalo O, de Miguel D, Ramirez-Labrada A, Martinez-Lostao L, Anel A, Pardo J, Marzo I and Naval J: Inhibition of autophagy with chloroquine potentiates carfilzomib-induced apoptosis in myeloma cells in vitro and in vivo. Cancer Lett 382: 1-10, 2016.

17 Liang DH, Choi DS, Ensor JE, Kaipparettu BA, Bass BL and Chang JC: The autophagy inhibitor chloroquine targets cancer stem cells in triple negative breast cancer by inducing mitochondrial damage and impairing DNA break repair. Cancer Lett 376: 249-258, 2016.

18 Gewirtz DA: The four faces of autophagy: implications for cancer therapy. Cancer Res 74: 647-651, 2014.

19 Yan C, Luo L, Goto S, Urata Y, Guo CY, Doi H, Kitazato K and Li TS: Enhanced autophagy in colorectal cancer stem cells does not contribute to radio-resistance. Oncotarget 7: 45112-45121, 2016.

20 Pickrell AM and Youle RJ: The roles of PINK1, parkin, and mitochondrial fidelity in Parkinson's disease. Neuron 85: 257273, 2015.

21 Redmann M, Dodson M, Boyer-Guittaut M, Darley-Usmar V and Zhang J: Mitophagy mechanisms and role in human diseases. Int J Biochem Cell Biol 53: 127-133, 2014.

22 Guo W, Sun Y, Liu W, Wu X, Guo L, Cai P, Wu X, Wu X, Shen Y, Shu Y, Gu Y and Xu Q: Small molecule-driven mitophagymediated NLRP3 inflammasome inhibition is responsible for the prevention of colitis-associated cancer. Autophagy 10: 972-985, 2014

23 Prasad S, Gupta SC and Tyagi AK: Reactive oxygen species (ROS) and cancer: Role of antioxidative nutraceuticals. Cancer Lett 387: 95-105, 2017.

24 Sentelle RD, Senkal CE, Jiang W, Ponnusamy S, Gencer S, Selvam SP, Ramshesh VK, Peterson YK, Lemasters JJ, Szulc ZM, Bielawski $\mathrm{J}$ and Ogretmen B: Ceramide targets autophagosomes to mitochondria and induces lethal mitophagy. Nat Chem Biol 8: 831-838, 2012.

25 Zhou J, Li G, Zheng Y, Shen HM, Hu X, Ming QL, Huang C, Li $\mathrm{P}$ and Gao N: A novel autophagy/mitophagy inhibitor liensinine sensitizes breast cancer cells to chemotherapy through DNM1L-mediated mitochondrial fission. Autophagy 11: 12591279, 2015.

26 Dany M and Ogretmen B: Ceramide induced mitophagy and tumor suppression. Biochim Biophys Acta 1853: 2834-2845, 2015.

27 Dany M, Gencer S, Nganga R, Thomas RJ, Oleinik N, Baron KD, Szulc ZM, Ruvolo P, Kornblau S, Andreeff $M$ and Ogretmen B: Targeting FLT3-ITD signaling mediates ceramidedependent mitophagy and attenuates drug resistance in AML. Blood 128: 1944-1958, 2016.
28 Lee Y, Lee HY, Hanna RA and Gustafsson AB: Mitochondrial autophagy by BNIP3 involves DRP1-mediated mitochondrial fission and recruitment of Parkin in cardiac myocytes. Am J Physiol Heart Circ Physiol 301: H1924-1931, 2011.

29 Kindler T, Lipka DB and Fischer T: FLT3 as a therapeutic target in AML: still challenging after all these years. Blood 116: 50895102, 2010.

30 Stein EM and Tallman MS: Emerging therapeutic drugs for AML. Blood 127: 71-78, 2016.

31 Mauro-Lizcano M, Esteban-Martinez L, Seco E, Serrano-Puebla A, Garcia-Ledo L, Figueiredo-Pereira C, Vieira HL and Boya P: New method to assess mitophagy flux by flow cytometry. Autophagy 11: 833-843, 2015.

32 Radogna F, Cerella C, Gaigneaux A, Christov C, Dicato M and Diederich M: Cell type-dependent ROS and mitophagy response leads to apoptosis or necroptosis in neuroblastoma. Oncogene 35: 3839-3853, 2016.

33 Luanpitpong S, Chanvorachote P, Nimmannit U, Leonard SS, Stehlik C, Wang L and Rojanasakul Y: Mitochondrial superoxide mediates doxorubicin-induced keratinocyte apoptosis through oxidative modification of ERK and BCL-2 ubiquitination. Biochem Pharmacol 83: 1643-1654, 2012.

34 Zhang S, Liu X, Bawa-Khalfe T, Lu LS, Lyu YL, Liu LF and Yeh ET: Identification of the molecular basis of doxorubicininduced cardiotoxicity. Nat Med 18: 1639-1642, 2012.

35 Jangamreddy JR, Ghavami S, Grabarek J, Kratz G, Wiechec E, Fredriksson BA, Rao Pariti RK, Cieslar-Pobuda A, Panigrahi S and Los MJ: Salinomycin induces activation of autophagy, mitophagy and affects mitochondrial polarity: differences between primary and cancer cells. Biochim Biophys Acta 1833: 2057-2069, 2013.

36 Hyttinen JM, Niittykoski M, Salminen A and Kaarniranta K: Maturation of autophagosomes and endosomes: a key role for RAB7. Biochim Biophys Acta 1833: 503-510, 2013.
Received November 2, 2017

Revised November 21, 2017

Accepted November 23, 2017 